\title{
Further evidence that mutations in INS can be a rare cause of Maturity-Onset Diabetes of the Young (MODY)
}

\author{
Trine W Boesgaard ${ }^{1 *}$, Stepanka Pruhova², Ehm A Andersson¹, Ondrej Cinek², Barbora Obermannova², \\ Jeannet Lauenborg ${ }^{3}$, Peter Damm ${ }^{3,4}$, Regine Bergholdt ${ }^{1}$, Flemming Pociot ${ }^{1}$, Charlotta Pisinger ${ }^{5}$, Fabrizio Barbetti ${ }^{6}$, \\ Jan Lebl' ${ }^{2}$, Oluf Pedersen ${ }^{1,7,8}$, Torben Hansen ${ }^{1,9}$
}

\begin{abstract}
Background: Insulin gene (INS) mutations have recently been described as a common cause of permanent neonatal diabetes (PNDM) and a rare cause of diabetes diagnosed in childhood or adulthood.

Methods: INS was sequenced in 116 maturity-onset diabetes of the young (MODYX) patients $(n=48$ Danish and $n=68$ (zech), 83 patients with gestational diabetes mellitus (GDM), 34 type 1 diabetic patients screened negative for glutamic acid decarboxylase (GAD), and 96 glucose tolerant individuals. The control group was randomly selected from the population-based sampled Inter99 study.

Results: One novel heterozygous mutation c.17G $>$ A, R6H, was identified in the pre-proinsulin gene (INS) in a Danish MODYX family. The proband was diagnosed at 20 years of age with mild diabetes and treated with diet and oral hypoglycaemic agent. Two other family members who carried the INS R6H were diagnosed with diabetes when 51 years old and with GDM when 27 years old, respectively. A fourth mutation carrier had normal glucose tolerance when 20 years old. Two carriers of INS R6H were also examined twice with an oral glucose tolerance test (OGTT) with 5 years interval. They both had a $~ 30 \%$ reduction in beta-cell function measured as insulinogenic index. In a Czech MODYX family a previously described R46Q mutation was found. The proband was diagnosed at 13 years of age and had been treated with insulin since onset of diabetes. Her mother and grandmother were diagnosed at 14 and 35 years of age, respectively, and were treated with oral hypoglycaemic agents and/or insulin.
\end{abstract}

Conclusion: Mutations in INS can be a rare cause of MODY and we conclude that screening for mutations in INS should be recommended in MODYX patients.

\section{Background}

Insulin gene (INS) mutations have recently been described as a common cause of permanent neonatal diabetes (PNDM) and a rare cause of diabetes diagnosed in childhood or adulthood [1-4]. Heterozygous mutations in INS account for $15-20 \%$ of cases of PNDM $[4,5]$. Gene discovery can lead to recognition of novel phenotypes [6] and recognition of novel clinical subgroups. For example, MODY was initially clinically defined as autosomal dominantly inherited, non insulin dependent, early-onset diabetes, but now there are at least eight distinct genetic subgroups of MODY, most of

\footnotetext{
* Correspondence: tweb@hagedorn.dk

${ }^{1}$ Steno Diabetes Centre and Hagedorn Research Institute, Gentofte, Denmark
}

which have a discrete phenotype and specialized treatment needs [6]. An R46Q INS mutation was recently described in a Norwegian study of 62 probands fulfilling conventional MODY criteria. In addition, they examined 223 patients from the population-based Norwegian Childhood Diabetes Registry and found an R55C INS mutation. One hundred blood donors were screened negative for these mutations [7]. The Italian study group on early onset diabetes has detected two INS mutations (A23S and G23S) in children negative for 5 type 1 diabetes (T1D) autoantibodies [1]. In addition, an R6C mutation was identified in an English MODY family and a L68M mutation was described in a family of Turkish origin with young-onset type 2 diabetes [3]. 
Furthermore, a recent screening for INS mutations in 252 patients diagnosed clinically with T1D between 6 months and 17 years of age identified 2 de novo heterozygous mutations G32S and R89C among the 25 (8\%) antibody-negative patients [8]. To our knowledge, no studies have screened women with gestational diabetes mellitus (GDM) for INS mutation. GDM was defined as an abnormal glucose tolerance diagnosed for the first time in pregnancy.

We aimed to evaluate the prevalence and the diseaseassociated phenotype of INS mutations among diabetic patients diagnosed with MODY, anti-body negative T1D or GDM.

\section{Methods}

INS was sequenced in 116 unrelated MODYX probands: 48 Danish, age at diagnosis (mean \pm SD) $24 \pm 19$ years, BMI $24.8 \pm 5.5 \mathrm{~kg} / \mathrm{m}^{2}$, and $68 \mathrm{Czech}$, age at diagnosis $18 \pm 8$ years, BMI $22.6 \pm 5.0 \mathrm{~kg} / \mathrm{m}^{2}, 83$ Danish diabetic patients previously diagnosed with GDM, age at examination $40 \pm 7$ years, BMI at examination $28.1 \pm 6.5 \mathrm{~kg} / \mathrm{m}^{2}$, 34 GAD autoantibody-negative T1D patients, age at diagnosis $20 \pm 16$ years and 96 glucose tolerant control individuals, age at examination $46 \pm 7$ years, BMI $26.4 \pm$ $4.4 \mathrm{~kg} / \mathrm{m}^{2}$. All MODYX probands were from families fulfilling the conventional criteria of MODY [5] defined by: diabetes diagnosed before 25 years of age in at least one of the family members. No treatment with insulin and/or measurable C-peptide at least one year after diagnosis. Autosomal dominant inherited diabetes with known diabetes in at least two consecutive generations.

All MODY probands were screened negative for mutations in the MODY genes HNF4A, GCK and HNF1A. Women with GDM and all GAD autoantibody-negative T1D patients had a positive family history of diabetes including a diabetic parents and or child with diabetes. The control group was randomly selected from the population-based study Inter99 [9]. Additional family members of the probands with an INS mutation were examined and screened for the family-specific INS mutation. The participants and examined family members gave their informed written consent, and the study protocols were approved by the ethical committees. The genomic sequence of INS was analysed in two segments covering the two exons, exon-intron boundaries and UTRs. Sequencing analyses were performed as described [4]. Analyses of the INS sequence revealed four lowfrequency (minor allele frequency $=0.05$ ) and three frequent (minor allele frequency $>0.05$ ) variants (Table 1 ).

\section{Results and Discussion}

One novel mutation c.17G>A, R6H, was identified in INS in a Danish MODYX family (Figure 1A). The mutation is located in the signal peptide of proinsulin and was not present in 96 control individuals. The proband, M132-1, had been diagnosed at 20 years of age with mild diabetes treated with diet and oral hypoglycaemic agent (OHA). His 20-years-old son, M132-2, carried the mutation but had normal glucose tolerance. The brother of the proband, M132-4, carried the mutation and had been diagnosed with diabetes at 51 years of age. A daughter, M132-5, of the brother carried the mutation and had been diagnosed with IGT at the age of 26 and with GDM at the age of 27 years. Two carriers of INS R6H, M132-2 and M132-4, were also examined with an OGTT at age 15 years and 46 years, respectively. Interestingly, they both had a $\sim 30 \%$ reduction in beta-cell function over a period of 5 years measured as insulinogenic index calculated from OGTT data (s-insulin 30 $\min (\mathrm{pmol} / \mathrm{l})$ - s-insulin $0 \mathrm{~min}(\mathrm{pmol} / \mathrm{l}) / \mathrm{p}$-glucose 30 $\min (\mathrm{mmol} / \mathrm{l})$. Insulinogenic index of M132-2 declined from 50 to 33 and for M132-4 from 19 to 13. BMI for M132-2 increased from 19.7 to $24.8 \mathrm{~kg} / \mathrm{m}^{2}$; for M132-4 BMI was unchanged, $24.2 \mathrm{~kg} / \mathrm{m}^{2}$, over the 5 -year period.

Table 1 INS gene variants identified in MODY $(n=116)$, T1DM $(n=34)$, GDM $(n=83)$ and controls $(n=96)$

\begin{tabular}{|c|c|c|c|c|c|c|c|}
\hline & & & & & MAF (\%) & & \\
\hline rs number & SNP & Position on chr. 11 & Part of gene & $\begin{array}{c}\text { MODY } \\
(n=116)\end{array}$ & $\begin{array}{l}\text { T1D } \mathrm{ab}^{-} \\
(n=34)\end{array}$ & $\begin{array}{c}\text { GDM } \\
(n=83)\end{array}$ & $\begin{array}{l}\text { Controls } \\
(n=92)\end{array}$ \\
\hline Novel & C. $-218 A>C$ & 2.138 .995 & 5'UTR & 0.4 & 0 & 0 & 0 \\
\hline rs689 & c. $-23 A>T$ & 2.138 .800 & Intron & 27 & 10 & 33 & 28 \\
\hline rs5505 & C. $-9 C>T$ & 2.138 .786 & 5'UTR & 0.9 & 0 & 0.6 & $\mathrm{O}^{\mathrm{A}}$ \\
\hline Novel & c.17G>A, p.R6H & 2.138 .761 & Exon & 0.4 & 0 & 0 & 0 \\
\hline Novel & c.137 G>A, p.R46Q & 2.138 .641 & Exon & 0.4 & 0 & 0 & 0 \\
\hline rs3842752 & $c{ }^{*} 9 \mathrm{C}>\mathrm{T}$ & 2.137 .649 & 3'UTR & 20 & 7 & 27 & 21 \\
\hline rs3842753 & $c^{*} 22 C>A$ & 2.137 .636 & $3^{\prime} U T R$ & 26 & 10 & 34 & 28 \\
\hline
\end{tabular}

SNP locations (-strand) are displayed counting from the first translated nucleotide in pre-proinsulin (INS). Base-pair positions are displayed counting from the p-arm telomere of chromosome 11 (according to the Base position feature in the Human (Homo sapiens) Genome Browser Gateway Human Mar. 2006 [hg18] assembly (http://genome.ucsc.edu/cgi-bin/hgGateway, assessed 19 June 2009). Minor allele frequencies (MAF) are given as a percentage.

A) Subsequent genotyping of 198 population-based individuals revealed a MAF of $0.7 \%$

Control individuals were collected as a subset of the population based study Inter99 of middle-aged Danish individuals. 


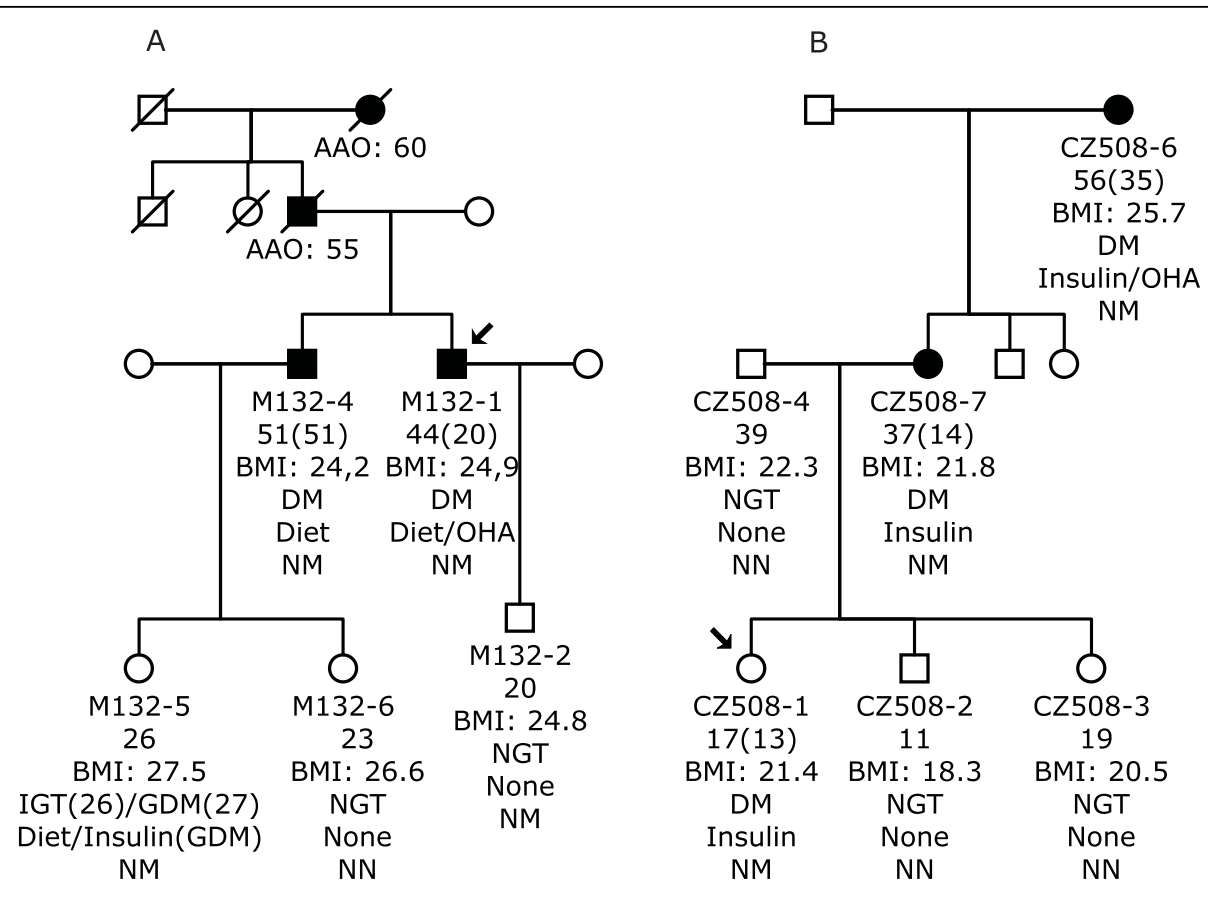

Figure 1 Segregation of INS R6H (Figure 1A) and R46Q (Figure 1B) mutations in two MODY families. Symbols denote the following: square, male; circle, female; empty symbol, normal glucose tolerant subject; filled symbol, diabetic person; quarter filled, impaired glucose tolerance status/gestational diabetes; symbol with arrowhead, proband. The text, under each individual represents the following: Subject id; age at examination (age at diagnosis); BMI ( $\mathrm{kg} / \mathrm{m}^{2}$ ); NGT, normal glucose tolerance; IGT, impaired glucose tolerance; GDM, gestational diabetes mellitus; DM, diabetes mellitus; treatment and mutation status; AAO, age of onset.

The R6 residue is conserved across mammalian species (rhesus monkey, elephant, mouse, dog and horse) and xenopus but is not conserved in lizard (S), platus $(G)$ or stikleback (Q). A mutation in the same codon, R6C, was recently described in an English MODY family affecting the proband, the mother and the maternal grandmother [3], underlining a putative importance of this residue for proper function of the signal peptide.

In a Czech MODY proband a previously described R46Q mutation was found (Figure 1B). The mutation is located at residue 22 of the $\mathrm{B}$ chain. The mutation cosegregates with diabetes in two affected relatives and is not present in any of the non-diabetic family members or 96 controls. The proband, a 17-year old girl, was diagnosed with diabetes at 13 years of age and had been treated with insulin since onset of disease. Her mother was diagnosed at 14 years of age with polyuria and polydipsia, and had been treated with insulin since onset of diabetes. The grandmother was diagnosed with diabetes at 35 years of age and treated with OHA and insulin. No coding mutations were found in GDM women or GAD-negative type 1 diabetic patients.

It is very likely that the $\mathrm{R} 46 \mathrm{Q}$ and $\mathrm{R} 6 \mathrm{H}$ mutations are disease causing and not just rare neutral polymorphisms. In already published studies $[1,3,7,8]$ a total of 1560 individuals primarily of Northern European ancestry have been screened for INS mutations. The R46Q mutation was identified in one Norwegian MODY family [7]. The mutation segregated with diabetes in the family. The R6H mutation has not been identified in the 1560 examined individuals indicating that this mutation is not a rare polymorphism. Furthermore, we have screened additional 74 Danish individuals (data not shown) without identifying the R46Q and R6H mutations.

\section{Conclusion}

Mutations in INS can be a rare cause of MODY and we conclude that screening for mutations in INS should be recommended in MODYX patients.

\section{Abbreviations}

GAD: Glutamic acid decarboxylase; GDM: Gestational diabetes mellitus; INS: Insulin gene; MODY: Maturity-onset diabetes of the young; PNDM: Permanent neonatal diabetes mellitus.

\section{Acknowledgements}

The study was supported by the Danish Medical Research Council and the Danish Diabetes Association. The authors wish to thank A. Forman, I.-L. Wantzin and M. Stendal for technical assistance, G. Lademann for secretarial support, A. L. Nielsen for database management, M.M.H. Kristensen for grant management, and the members of the Inter99 team. The authors from the Czech Republic were supported by a grant "Monogenic diabetes in 
childhood: from genetic to therapy" from Norway though the Norwegian Financial Mechanism (No CZ0100).

\section{Author details}

'Steno Diabetes Centre and Hagedorn Research Institute, Gentofte, Denmark. ${ }^{2}$ Department of Pediatrics, Second Faculty of Medicine, Charles University, Prague, Czech Republic. ${ }^{3}$ Center for Pregnant Women with Diabetes, Department of Obstetrics, Copenhagen University Hospital, Rigshospitalet, Denmark. ${ }^{4}$ Faculty of Health Science, University of Copenhagen, Copenhagen, Denmark. ${ }^{5}$ Research Centre for Prevention and Health, Glostrup University Hospital, Glostrup, Denmark. ${ }^{6}$ Endocrinology and Diabetes Unit, Department of Paediatric Medicine, Bambino Gesù Children's Hospital IRCCS, Piazza S. Onofrio 4, Rome, Italy. Institute of Biomedical Science, Faculty of Health Sciences, University of Copenhagen, Copenhagen, Denmark. ${ }^{8}$ Faculty of Health Sciences, University of Aarhus, Aarhus, Denmark. ${ }^{9}$ Faculty of Health Sciences, University of Southern Denmark, Denmark.

\section{Authors' contributions}

The concept and idea regarding the study belong to EAA, TWEB, OP and $\mathrm{TH}$. The collection of study subjects was planned and performed by SP, OC, $B O, J L, J L, P D, R B, F P, C P, T W E B, O P$ and $T H$.

The original hypothesis regarding the genetic study was conceived by EAA and TWEB, and approved by OP and TH. Detail planning of analyses and study design was performed by EAA and TWEB, and approved by FB, OP and TH. EAA, TWEB, OP and TH contributed to the establishment of study population databases specific for this study. Statistical analyses were performed by EA and TWEB. The first manuscript was written by TWEB and EAA (equal contributions) and the final draft was produced by TWEB, OP and $\mathrm{TH}$. All authors revised the manuscript and contributed to the discussion.

\section{Competing interests}

T.W Boesgaard, E.A Andersson, R Bergholdt, F Pociot, O Pedersen and T Hansen hold stock in Novo Nordisk, and O Pedersen and T Hansen have received lecture fees from pharmaceutical companies. All other authors declare that there is no competing interest associated with this manuscript.

\section{Received: 13 August 2009 Accepted: 12 March 2010}

Published: 12 March 2010

\section{References}

1. Bonfanti R, Colombo C, Nocerino V, et al: Insulin gene mutations as cause of diabetes in children negative for five type 1 diabetes autoantibodies. Diabetes care 2009, 32:123-125.

2. Colombo C, Porzio O, Liu M, et al: Seven mutations in the human insulin gene linked to permanent neonatal/infancy-onset diabetes mellitus. $J$ Clin Invest 2008, 118:2148-2156.

3. Edghill EL, Flanagan SE, Patch AM, et al: Insulin mutation screening in 1,044 patients with diabetes: mutations in the INS gene are a common cause of neonatal diabetes but a rare cause of diabetes diagnosed in childhood or adulthood. Diabetes 2008, 57:1034-1042.

4. Støy J, Edghill EL, Flanagan SE, et al: Insulin gene mutations as a cause of permanent neonatal diabetes. Proceedings of the National Academy of Sciences 2007, 104:15040-15044

5. Murphy R, Ellard S, Hattersley AT: Clinical implications of a molecular genetic classification of monogenic beta-cell diabetes. Nat Clin Pract Endocrinol Metab 2008, 4:200-213.

6. McCarthy MI, Hattersley AT: Learning from molecular genetics: novel insights arising from the definition of genes for monogenic and type 2 diabetes. Diabetes 2008, 57:2889-2898.

7. Molven A, Ringdal M, Nordbo AM, et al: Mutations in the Insulin Gene Can Cause MODY and Autoantibody-Negative Type 1 Diabetes. Diabetes 2008, 57:1131-1135.

8. Rubio-Cabezas O, Edghill EL, Argente J, Hattersley AT: Testing for monogenic diabetes among children and adolescents with antibodynegative clinically defined Type 1 diabetes. Diabet Med 2009, 26:1070-1074

9. Jørgensen T, Borch-Johnsen $\mathrm{K}$, Thomsen TF, Ibsen H, Glümer C, Pisinger C: A randomized non-pharmacological intervention study for prevention of ischaemic heart disease: baseline results Inter99. Eur $J$ Cardiovasc Prev Rehabil 2003, 10:377-386.

\section{Pre-publication history}

The pre-publication history for this paper can be accessed here:http://www. biomedcentral.com/1471-2350/11/42/prepub

doi:10.1186/1471-2350-11-42

Cite this article as: Boesgaard et al:: Further evidence that mutations in INS can be a rare cause of Maturity-Onset Diabetes of the Young (MODY). BMC Medical Genetics 2010 11:42.

\section{Submit your next manuscript to BioMed Central and take full advantage of:}

- Convenient online submission

- Thorough peer review

- No space constraints or color figure charges

- Immediate publication on acceptance

- Inclusion in PubMed, CAS, Scopus and Google Scholar

- Research which is freely available for redistribution

Submit your manuscript at www.biomedcentral.com/submit
C Biomed Central 